\title{
Doxycycline and Sitafloxacin Combination Therapy for Treating Highly Resistant Mycoplasma genitalium
}

Duygu Durukan, Michelle Doyle, Gerald Murray, Kaveesha Bodiyabadu, Lenka Vodstrcil, Eric P.F. Chow, Jorgen S. Jensen, Christopher K. Fairley, Ivette Aguirre, Catriona S. Bradshaw

Antimicrobial-resistant Mycoplasma genitalium is becoming increasingly common and creating major treatment challenges. We present early data on combination therapy with doxycycline and sitafloxacin to treat highly resistant $M$. genitalium. We found the regimen was well tolerated and cured 11/12 infections that had failed prior regimens with moxifloxacin and pristinamycin.

M ycoplasma genitalium is a sexually transmitted bacterium with marked capacity for developing antimicrobial resistance (1). Macrolides and 4th-generation fluroquinolones, such as moxifloxacin, have been the main agents displaying efficacy against $M$. genitalium. However, macrolide resistance has increased to $>50 \%$ in many nations, and quinolone resistance is increasing (2-6). In Australia, $16 \%$ of M. genitalium strains are reported to have dual-class resistance (5), and Japan reports dual-class resistance of up to $25 \%$ (2), resulting in infections that often cannot be cured with current recommended therapies.

Sequential monotherapy with doxycycline followed by moxifloxacin (7-9) is currently first-line therapy for macrolide-resistant $M$. genitalium in guidelines in Australia and the United Kingdom and achieves cure in $92 \%$ of cases $(95 \%$ CI $88.1 \%-94.6 \%)$ at our service (7). When the doxycycline/moxifloxacin

Author affiliations: Alfred Health, Carlton, Victoria, Australia

(D. Durukan, M. Doyle, L. Vodstrcil, E.P.F. Chow, C.K. Fairley,

I. Aguirre, C.S. Bradshaw); Monash University, Clayton, Victoria,

Australia (D. Durukan, L. Vodstrcil, E.P.F. Chow, C.K. Fairley,

C.S. Bradshaw); Royal Children's Hospital, Parkville, Victoria,

Australia (G. Murray, K. Bodiyabadu); The Royal Women's

Hospital, Parkville (G. Murray, K. Bodiyabadu); Statens Serum

Institut, Copenhagen, Denmark (J.S. Jensen)

DOI: https://doi.org/10.3201/eid2608.191806 sequential regimen fails, we use a pristinamycinbased regimen, which achieves $75 \%$ cure $(95 \%$ CI $66 \%-82 \%$ ) (10). Since August 2017, for patients in whom both regimens failed, we administered a combination of $100 \mathrm{mg}$ doxycycline and $100 \mathrm{mg}$ sitafloxacin 2 times/day for 7 days.

Access to sitafloxacin is limited in many countries, but it is available in the Asia-Pacific region. Most publications on sitafloxacin are from Japan, where its use as a monotherapy is reported to cure $\approx 90 \%$ of $M$. genitalium infections (11). However, combination therapies can optimize cure and prevent further resistance in bacteria prone to developing resistance, such as $M$. genitalium. In vitro, a combination of doxycycline and sitafloxacin (doxycycline+sitafloxacin) shows synergy for quinolone-susceptible $M$. genitalium strains but has not been evaluated for highly resistant strains (J.S. Jensen, unpub. data). We provide early data on the efficacy and tolerability of a 7-day doxycycline+sitafloxacin combination therapy for treatment-resistant $M$. genitalium. The ethics committee of Alfred Hospital (Melbourne) approved this study (approval no. 232/16).

\section{The Study}

The study included 12 cases of macrolide-resistant $M$. genitalium detected among all patients assessed with the clinical protocol at Melbourne Sexual Health Centre (MSHC), Carlton, Victoria, Australia, for routine M. genitalium testing $(7,9)$ during August 2017-April 2019 (Figure). During the study period, 96 (8\%) of $M$. genitalium cases failed to respond to doxycycline/ moxifloxacin; we subsequently treated 56 with pristinamycin, which failed in $15(27 \%)$ patients. All 15 opted for combination therapy; 11 provided a test of cure, and the other 4 did not complete follow up. One 


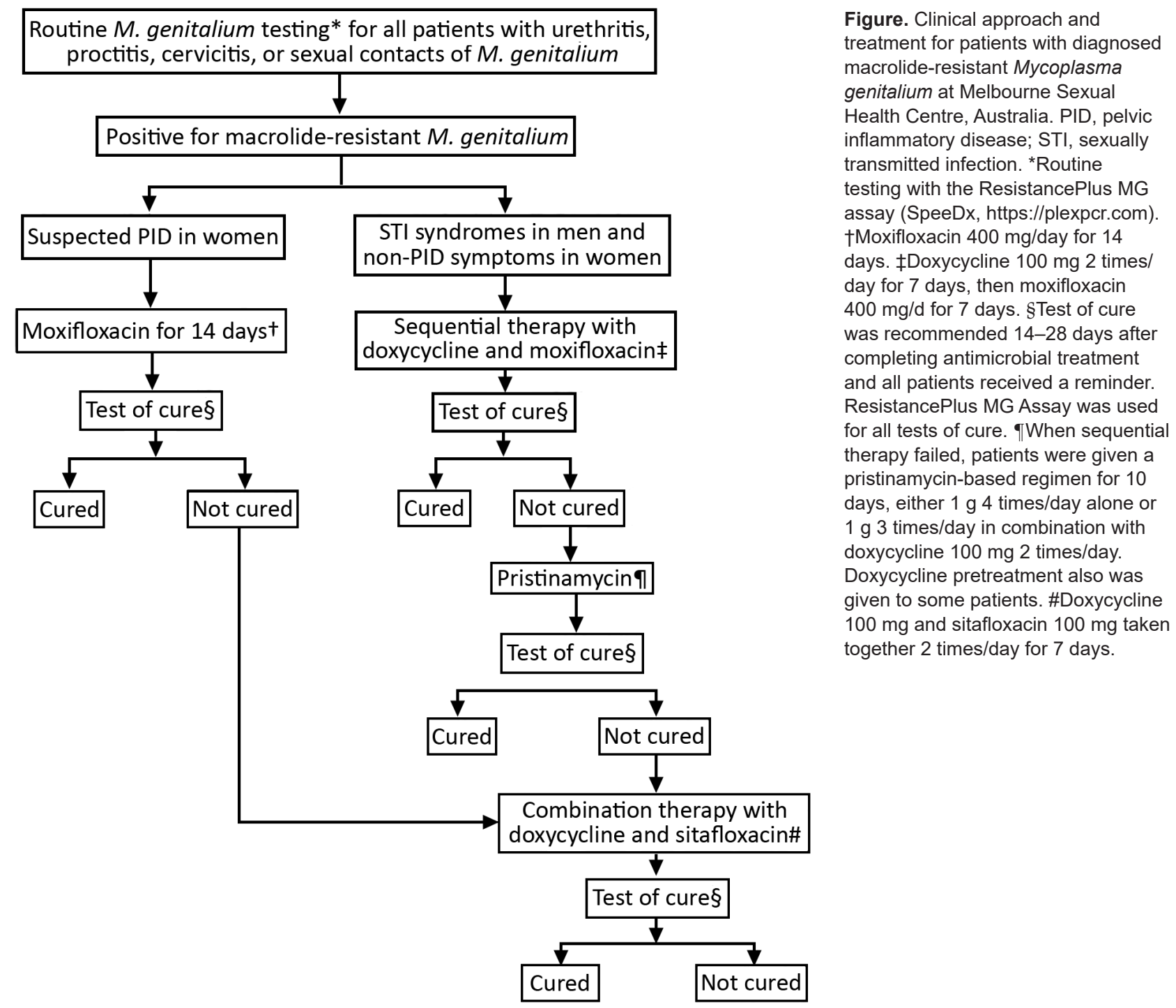

Figure. Clinical approach and treatment for patients with diagnosed macrolide-resistant Mycoplasma genitalium at Melbourne Sexual assay (SpeeDx, https://plexpcr.com). †Moxifloxacin $400 \mathrm{mg} /$ day for 14 days. $\ddagger$ Doxycycline $100 \mathrm{mg} 2$ times/ day for 7 days, then moxifloxacin $400 \mathrm{mg} / \mathrm{d}$ for 7 days. §Test of cure was recommended $14-28$ days after completing antimicrobial treatment and all patients received a reminder. RistancePlus MG Assay was used days, either $1 \mathrm{~g} 4$ times/day alone or $1 \mathrm{~g} 3$ times/day in combination with doxycycline $100 \mathrm{mg} 2$ times/day. Doxycycline pretreatment also was given to some patients. \#Doxycycline $100 \mathrm{mg}$ and sitafloxacin $100 \mathrm{mg}$ taken together 2 times/day for 7 days. additional patient also received combination therapy because her pelvic inflammatory disease (PID) did not respond to moxifloxacin. Our final analysis included 12 patients.

Among study participants, 9 sought treatment for urogenital symptoms and 1 for PID; 2 were asymptomatic contacts of persons with M. genitalium. Median age was 29 years (interquartile range [IQR] 27-32 years). All men (10/12) had urethral infections; the 2 women had cervicovaginal infections.

We tested patient samples using the ResistancePlus MG Assay (SpeeDx, https:/ / plexpcr.com). We defined treatment-resistant $M$. genitalium as microbiological failure and persistent symptoms after first-line and second-line therapies failed. We classified microbial cure as M. genitalium not detected and microbial failure as a positive result on test of cure 14-28 days after completing antimicrobial drug therapy.

At MSHC, we often give doxycycline before the main regimen to reduce $M$. genitalium load and optimize cure (12). We term moxifloxacin-containing regimens as first-line, pristinamycin-containing regimens as second-line, and combination therapy as third-line (Table 1). All 12 patients received a moxifloxacinbased regimen; 9 had sequential doxycycline/moxifloxacin in keeping with clinical guidelines (9). The other 3 had moxifloxacin alone, 1 for PID, and 2 received treatments prior to coming to MSHC (1 was treated for 10 days and the other for 30 days, but we do not know the physician's rationale for treatment duration). Moxifloxacin-based regimens failed in all patients; 11 were then treated with pristinamycin, which also failed. We administered combination 
Table 1. Antimicrobial regimens and test of cure data for patients treated for Mycoplasma genitalium with doxycycline and sitafloxacin combination therapy, Melbourne Sexual Health Centre, Carleton, Victoria, Australia*

\begin{tabular}{|c|c|c|c|c|c|c|c|c|c|c|}
\hline \multirow[b]{2}{*}{ Case no. } & \multirow{2}{*}{$\begin{array}{c}\text { Baseline } \\
\text { test }\end{array}$} & \multicolumn{2}{|c|}{$\begin{array}{l}\text { First-line therapy, } \\
\text { sequential; d }\end{array}$} & \multirow[b]{2}{*}{ TOC } & \multicolumn{2}{|c|}{$\begin{array}{l}\text { Second-line therapy, } \\
\text { sequential; d }\end{array}$} & \multirow[b]{2}{*}{ TOC } & \multicolumn{2}{|c|}{$\begin{array}{l}\text { Third-line therapy, } \\
\text { combination; d }\end{array}$} & \multirow[b]{2}{*}{ TOC } \\
\hline & & Doxy† & Moxi & & Doxy† & Pris & & Doxy† & Combination $\ddagger$ & \\
\hline $1 \S$ & + & 7 & 7 & + & 7 & 10 & + & 21 & 7 & Cured \\
\hline 2 & + & 7 & 7 & + & 7 & 10 & + & 28 & 7 & Cured \\
\hline 3 & + & 7 & 7 & + & 7 & 10 & + & 21 & 7 & Cured \\
\hline $4 \S$ & + & None & 10ा & + & 7 & 10 & + & None & 7 & Cured \\
\hline 5 & + & 7 & 7 & + & 7 & 10 & + & None & 7 & Cured \\
\hline $6 \S$ & + & None & $30 \pi$ & + & 21 & 10 & + & 14 & 7 & Cured \\
\hline 7 & + & 7 & 7 & + & None & 10 & + & 7 & 7 & Cured \\
\hline 8 & + & 7 & 7 & + & None & 10 & + & 7 & 7 & Cured \\
\hline 9 & + & 7 & 7 & + & 7 & 10 & + & 7 & 7 & Failed \\
\hline $10 \S$ & + & 7 & 7П & + & None & 10 & + & 3 & 7 & Cured \\
\hline $11 \S$ & + & None & $14 \#$ & + & None ${ }^{\star *}$ & None & None & 5 & 7 & Cured \\
\hline 12 & + & 7 & 7 & + & $10 \dagger \dagger$ & 10 & + & 3 & 7 & Cured \\
\hline
\end{tabular}

*Doxy, doxycycline; Moxi, moxifloxacin; Pris, pristinamycin; TOC, test of cure; +, macrolide-resistant $M$. genitalium detected.

†Doxycycline $100 \mathrm{mg} 2$ times/day commonly was given first as monotherapy in first, second, and third drug regimens; duration is specified for each case. †Sitafloxacin $100 \mathrm{mg} 2$ times/day and doxycycline $100 \mathrm{mg} 2$ times/day were given concurrently for 7 days.

§Patients who had prior failed antimicrobial therapy for M. genitalium infection before coming to Melbourne Sexual Health Centre (MSHC). Case no. 1 received $1 \mathrm{~g}$ azithromycin before coming to $\mathrm{MSHC}$ and this regimen failed. Case no. 4 received moxifloxacin $400 \mathrm{mg} / \mathrm{d}$ for 10 days before coming to MSHC and this regimen failed. Case no. 6 received $31 \mathrm{~g}$ doses of azithromycin given on separate occasions and this regimen failed, then received 2 courses of doxycycline $100 \mathrm{mg} 2$ times/day for $14 \mathrm{~d}$ each which also failed; then received a 30 -day course of moxifloxacin $400 \mathrm{mg} / \mathrm{d}$ which also failed, before coming to MSHC. Case no. 10 received doxycycline $100 \mathrm{mg} 2$ times/day for $7 \mathrm{~d}$ then $1 \mathrm{~g}$ azithromycin which failed; then received doxycycline 100 $\mathrm{mg} 2$ times/day for $7 \mathrm{~d}$ which failed, after which the patient received moxifloxacin $400 \mathrm{mg} / \mathrm{d}$ for $7 \mathrm{~d}$, which also failed before coming to MSHC. Case no. 11 received doxycycline and azithromycin at unspecified doses or duration before coming to MSHC.

TMoxifloxacin-containing regimen given to a patient in the community before they came to MSHC. This regimen often varied from firstline therapy given at MSHC.

\#Moxifloxacin $400 \mathrm{mg} / \mathrm{d} \times 14 \mathrm{~d}$ was given as a first regimen to this patient because of diagnosed pelvic inflammatory disease.

${ }^{* *}$ Patient was diagnosed with possible $M$. genitalium-related pelvic inflammatory disease and did not receive a pristinamycin-containing regimen (14).

††Patient was given $1 \mathrm{~g}$ pristinamycin 3 times/day in combination with doxycycline $100 \mathrm{mg} 2$ times/day rather than $1 \mathrm{~g} 4$ times/day because both regimens have shown equivalent efficacy at our service (10).

therapy without preceding pristinamycin to 1 patient because of concerns regarding her PID.

All patients received doxycycline+sitafloxacin combination therapy for 7 days; 9 had preceding doxycycline for varying durations (Table 1). Among 12 patients, 11 (91.7\%; 95\% CI 64.9\%-98.5\%) were cured and achieved complete symptom resolution after combination therapy. Combination therapy failed in 1 patient who experienced persistent dysuria. Median time to test of cure after combination therapy was 20 (IQR 14-24) days. Median duration from first $M$. genitalium diagnosis to cure was 125 (IQR 106-144) days. Before test of cure, all patients were classified as no- or low-risk for reinfection by the treating clinician on the basis of no sex or $100 \%$ condom use with any partner or sex with a fully treated partner in the interval between treatment and test of cure $(7,12)$.

All patients whose first-line and second-line therapies failed were symptomatic, including the 2 who initially were asymptomatic contacts. All 10 men reported persistent fluctuating dysuria, 6 reported urethral discharge, 2 urethral irritation or itching, and 1 meatal inflammation. Both women reported fluctuating abnormal vaginal discharge, 1 reported intermittent dysuria, and the patient with PID reported persistent dyspareunia. Men typically experienced a stepwise reduction in urethral symptoms after commencing antimicrobial drugs, but dysuria reemerged during follow-up.

Among 10 patients for whom adherence and adverse effects are available, 9 (90.0\%; 95\% CI 60.0\%$99.5 \%$ ) reported taking all doses of both drugs, including the patient whose treatment failed; 1 reported missing 1 tablet of sitafloxacin. Six $(60.0 \%$; $95 \%$ CI $31.3 \%-83.2 \%)$ patients reported no adverse effects. Among the other 4, adverse effects were mild and resolved spontaneously (1 each of diarrhea, arthralgia, tendon pain, and possible blurred vision).

Sanger sequencing of the quinolone resistancedetermining regions of the parC and gyrA genes revealed single nucleotide polymorphisms for parC in all cases and for gyrA in 5/12 cases before combination therapy (Table 2). The parC mutations corresponded to amino acid changes S83I (G248T; $\mathrm{n}=11$ ) and D87N (G259A; $\mathrm{n}=1)$. The gyrA mutations corresponded to amino acid changes M95I $(\mathrm{G} 285 \mathrm{~A} ; \mathrm{n}=$ 3), A79S (G235T; $n=1)$, and D99N (G295A; $n=1)$. In 1 case, a gyrA mutation appeared to develop after moxifloxacin failure (case 9; Table 2). The patient in whom combination therapy failed had a single parC S83I change detected (Table 2).

\section{Conclusions}

Combination therapy with doxycycline+sitafloxacin was well tolerated and effective against treatment- 
Table 2. Amino acid changes in the quinolone resistance-determining regions of parC and gyrA genes of macrolide-resistant Mycoplasma genitalium in patients treated with combination therapy, Melbourne Sexual Health Centre, Carlton, Victoria, Australia*

\begin{tabular}{|c|c|c|c|c|c|c|c|c|c|}
\hline \multirow{2}{*}{$\begin{array}{l}\text { Case } \\
\text { no. }\end{array}$} & \multirow{2}{*}{$\begin{array}{l}\text { Sexual } \\
\text { orientation }\end{array}$} & \multicolumn{2}{|c|}{ Baseline test } & \multicolumn{2}{|c|}{ TOC after first-line therapy } & \multicolumn{2}{|c|}{$\begin{array}{c}\text { TOC after second-line } \\
\text { therapy }\end{array}$} & \multicolumn{2}{|c|}{$\begin{array}{l}\text { TOC after third-line } \\
\text { therapy }\end{array}$} \\
\hline & & parC & gyrA & parC & gyrA & parC & gyrA & parC & gyrA \\
\hline 1 & MSM & NA & NA & S83I & $\mathrm{n} / \mathrm{A}$ & S83I & D99N & & \\
\hline 2 & MSM & S83I & M95I & S83I & M95I & S83I & M95I & & \\
\hline 3 & MSM & S83I & WT & S83I & WT & S83I & WT & & \\
\hline 4 & MSW & NA & NA & NA & NA & S83I & A79S & & \\
\hline 5 & MSW & D87N & WT & D87N & WT & D87N & WT & & \\
\hline 6 & MSW & NA & NA & S83I & WT & S83I & WT & & \\
\hline 7 & MSW & S83I & WT & S83I & WT & S83I & WT & & \\
\hline 8 & MSM & S83I & WT & S83I & WT & S83I & WT & & \\
\hline 9 & MSW & S83I & WT & S83I & WT & S83I & WT & S83I & WT \\
\hline 10 & MSM & NA & NA & S83I & WT & S83I & WT & & \\
\hline 11 & W & S83I & WT & S83I & M95I & ND & ND & & \\
\hline 12 & W & S83I & M95I & S83I & M95I & S83I & M95I & & \\
\hline
\end{tabular}

resistant $M$. genitalium. The regimen was acceptable to clinicians and is now used as our thirdline regimen.

Moxifloxacin failure has been associated with specific polymorphisms in the quinolone resistancedetermining regions of $\operatorname{parC}(2,5)$. The parC G248T mutation, which causes amino acid change S83I, is the most common mutation associated with moxifloxacin failure (5). The less common G259A(D87N) mutation has been associated with higher moxifloxacin MICs in $3 \mathrm{M}$. genitalium strains (J.S. Jensen, unpub. data). S83I contributes to both sitafloxacin and moxifloxacin failure (5). Although sitafloxacin is more likely than moxifloxacin to cure an infection carrying an S83I mutation, we previously found concurrent gyrA mutations, particularly M95I, increased the risk for sitafloxacin failure (5). In this study, moxifloxacin failed in all 12 cases with a parC mutation and 5 had a concurrent gyrA mutation. However, 11/12 cases were cured with the doxycycline+sitafloxacin combination. Of note, the 1 treatment failure occurred in a case with only the $\operatorname{parC}$ G248T/S83I mutation, no concurrent gyrA mutation, and no more detectable resistance than cured cases.

Of note, sitafloxacin alone might have cured some or all infections. Further studies comparing sequential and combination therapy with doxycyline+sitafloxacin in highly resistant $M$. genitalium are needed. However, treatment failures, particularly in cases with concurrent parC and gyrA mutations, would be expected. Moreover, the variable duration of preceding doxycycline may have improved cure.

In conclusion, our results provide important early data on the efficacy and tolerability of doxycycline+sitafloxacin combination therapy to cure highly resistant $M$. genitalium infections. This approach could become part of a broader stewardship strategy to evaluate combination therapy, which might be needed to further prevent development of antimicrobial-resistant M. genitalium.

\section{Acknowledgments}

We thank the staff at Melbourne Sexual Health Centre for collecting the clinical data for this study, Afrizal Afrizal and Jun Kit Sze for their help with maintaining our electronic patient database, and the laboratory staff at the Royal Women's Hospital for handling the specimens.

This work was supported by Monash University Central Clinical School departmental scholarship to D.D. and the National Health and Medical Research Council Early Career Fellowship to E.P.F.C. (no. 1091226).

G.M. reports holding joint grants with SpeeDx Pty. Ltd (https:// plexpcr.com) outside the submitted work. Melbourne Sexual Health Centre receives institutional funding from Speedx Pty Ltd for support in other research projects on Mycoplasma genitalium, in which C.S.B., M.D., and C.K.F. are involved. E.P.F.C. reports grants from Merck \& Co. (https:/ / www.merck.com) and Seqirus Australia (https:/ / www.seqirus.com) outside the submitted work. C.K.F. reports grants from SpeeDx Pty. Ltd. during the study. C.S.B. reports grants from SpeeDx Pty. Ltd. outside the submitted work. All other authors report no potential conflicts.

\section{About the Author}

Ms. Durukan is an MD-PhD candidate at Monash University, Clayton, Victoria, Australia. Her primary research interests are sexually transmitted infections and epidemiology. 


\section{References}

1. Bradshaw CS, Jensen JS, Waites KB. New horizons in Mycoplasma genitalium treatment. J Infect Dis. 2017; 216(suppl_2):S412-S9. https://doi.org/10.1093/infdis/jix132

2. Hamasuna R, Le PT, Kutsuna S, Furubayashi K, Matsumoto M, Ohmagari N, et al. Mutations in $\operatorname{ParC}$ and GyrA of moxifloxacin-resistant and susceptible Mycoplasma genitalium strains. PLoS One. 2018;13:e0198355.

https:/ / doi.org/10.1371/journal.pone.0198355

3. Braam JF, van Dommelen L, Henquet CJM, van de Bovenkamp JHB, Kusters JG. Multidrug-resistant Mycoplasma genitalium infections in Europe. Eur J Clin Microbiol Infect Dis. 2017;36:1565-7. https://doi.org/10.1007/ s10096-017-2969-9

4. Xiao L, Waites KB, Van Der Pol B, Aaron KJ, Hook EW III, Geisler WM. Mycoplasma genitalium infections with macrolide and fluoroquinolone resistance-associated mutations in heterosexual African American couples in Alabama. Sex Transm Dis. 2019;46:18-24. https:// doi.org/ 10.1097/OLQ.0000000000000891

5. Murray GL, Bodiyabadu K, Danielewski J, Garland SM, Machalek DA, Fairley CK, et al. Moxifloxacin and sitafloxacin treatment failure in Mycoplasma genitalium infection: sssociation with parC mutation G248T (S83I) and concurrent gyrA mutations. J Infect Dis. 2020;221:1017-1024. PubMed https://doi.org/10.1093/infdis/jiz550

6. Machalek D, Tao Y, Shilling H, Jensen J, Unemo M, Murray G, et al. P601 Macrolide and fluoroquinolone resistance-associated mutations in Mycoplasma genitalium: a systematic review and meta-analysis. Sex Transm Infect. 2019;95(Suppl 1):A266. https://doi.org/10.1136/sextrans-2019-sti.669

7. Durukan D, Read TRH, Murray G, Doyle M, Chow EPF, Vodstrcil LA, et al. Resistance-guided antimicrobial therapy using doxycycline-moxifloxacin and doxycycline- $2.5 \mathrm{~g}$ azithromycin for the treatment of Mycoplasma genitalium infection: efficacy and tolerability. Clin Infect Dis. 2019 Oct 20 [Epub ahead of print]. https://doi.org/10.1093/cid/ ciz10312019

8. Soni S, Horner P, Rayment M, Pinto-Sander N, Naous N, Parkhouse A, et al. British Association for Sexual Health and HIV national guideline for the management of infection with Mycoplasma genitalium (2018). Int J STD AIDS. 2019;30:938-50. https://doi.org/10.1177/0956462419825948

9. Australia Sexual Health Alliance. Australian STI management guidelines for use in primary care. Sydney, Australia: The Alliance; 2018 [cited 2019 Dec 3]. http:/ / www.sti.guidelines.org.au

10. Read TRH, Jensen JS, Fairley CK, Grant M, Danielewski JA, $\mathrm{Su}$ J, et al. Use of pristinamycin for macrolide-resistant Mycoplasma genitalium infection. Emerg Infect Dis. 2018;24:328-35. https://doi.org/10.3201/eid2402.170902

11. Takahashi S, Hamasuna R, Yasuda M, Ito S, Ito K, Kawai S, et al. Clinical efficacy of sitafloxacin $100 \mathrm{mg}$ twice daily for 7 days for patients with non-gonococcal urethritis. J Infect Chemother. 2013;19:941-5. https://doi.org/10.1007/ s10156-013-0620-y

12. Read TRH, Fairley CK, Murray GL, Jensen JS, Danielewski J, Worthington $\mathrm{K}$, et al. Outcomes of resistance-guided sequential treatment of Mycoplasma genitalium infections: a prospective evaluation. Clin Infect Dis. 2019;68:554-60. https:/ / doi.org/10.1093/cid/ciy477

Address for correspondence: Duygu Durukan, Melbourne Sexual Health Centre, 580 Swanston St, Carlton 3053, VIC, Australia; email: duygu.durukan@monash.edu

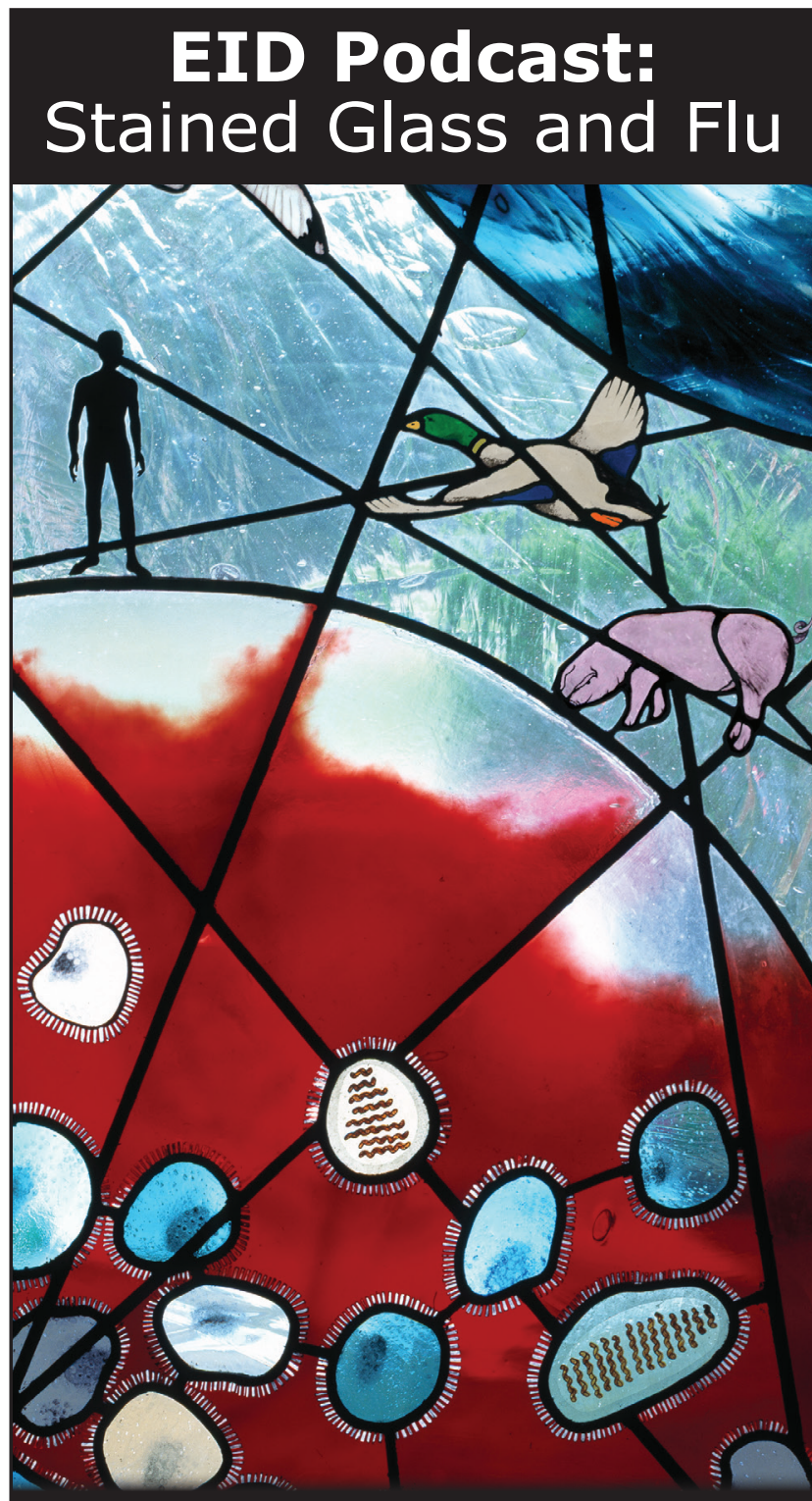

The work of art shown here depicts the interrelationship of human, animal, and environmental health.

Stained-glass windows have been appreciated for their utility and splendor for more than 1,000 years, and this engaging work of art by stained glass artist Jenny Hammond reminds us that influenza A viruses-which can be easily spread between animals and humans, use various host species, and exist in many different environments-remain an enduring and global health concern.

\section{Visit our website to listen:} EMERGING WFECTIOUS DISEASES https://www2c.cdc.gov/ podcasts/player. asp?f=8644950 